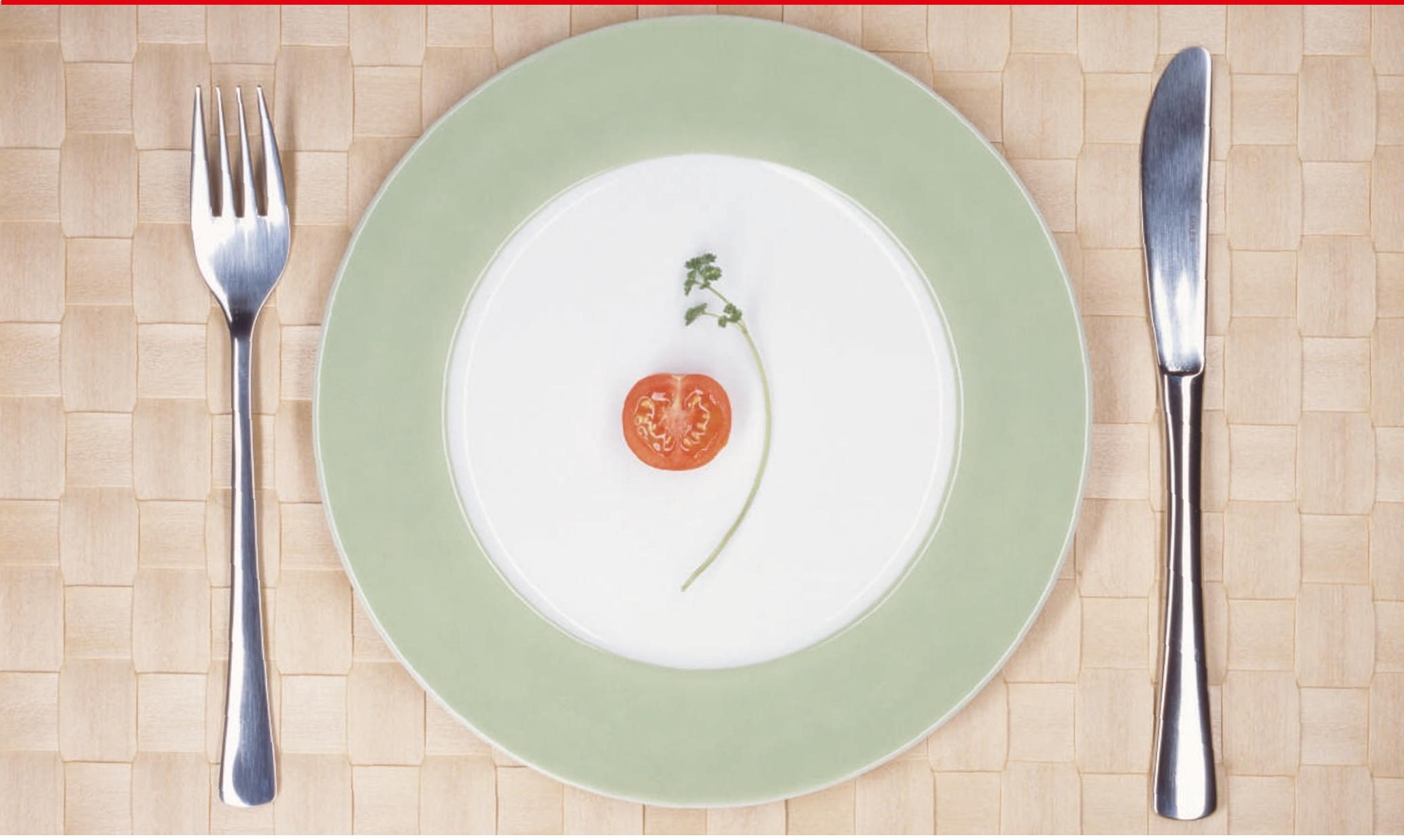

\title{
Eat your cake and have it
}

\section{Reducing your calorie intake makes you live longer - if you're a rat or a worm. Laura Spinney asks whether the same holds for humans - and if it does, whether the benefits could be put in a pill.}

t may be just a dot in the Pacific Ocean, but the Japanese island of Okinawa has long held a fascination for researchers into ageing. Its inhabitants include an unusually high number of centenarians, and fewer of its people die from diseases of old age such as cancer and stroke. Some have suggested the islanders' secret is their frugal diet. Now scientists are attempting to put this idea to the test - and are even developing drugs that they hope will produce the same effect. Could the path to a long and healthy life really be as easy as swallowing a tablet?

The issue divides researchers. Calorie restriction, or cutting energy intake below energy expenditure, can slow ageing, reduce mortality, and extend maximum lifespan in rats, mice, fish, flies, worms and yeast. But to date, the only evidence that it works in humans is anecdotal. A 1978 study of inhabitants of Okinawa ${ }^{1}$ showed that energy intake among adults was roughly $80 \%$ of the Japanese average. Some researchers have held this up as evidence that restricting diet reduces agerelated diseases and extends longevity. But gerontologist Sataro Goto of Toho University in Chiba, Japan, points out that Japanese people have a daily energy intake nearly $20 \%$ less than the average of developed countries, and that the mean life-expectancy of Japanese women is 85 years - not significantly more than that of women in all developed countries.

\section{Hungry for life}

This hasn't stopped thousands of people all over the world intentionally going hungry in the hope of living longer and healthier. But no one yet knows if their punishing lifestyle will pay off. So an experiment designed to find out is being watched closely by researchers into ageing.

The CALERIE (Comprehensive Assessment of Long-term Effects of Reducing Intake of Energy) study, sponsored by the US National Institute on Aging (NIA), published the outcome of its first phase in April ${ }^{2}$. Forty-eight men and women were randomly assigned to diets that would either maintain their weight, or reduce their calorie intake by up to $25 \%$ below the level required to maintain their weight. After six months, those on restricted diets showed lower body temperature and levels of insulin in their blood when they had not had a meal, both characteristics of long-lived people and animals. This slowing of the metabolism explains why people on calorierestricted diets don't starve to death: their body's energy requirements drop to meet the number of calories in their diet.

CALERIE is a departure in this hypeinfested field, because the volunteers are of normal weight or slightly overweight, but not obese. Most studies so far have used obese individuals, muddying the waters with the health benefits of reducing obesity. The second phase of the study will begin this summer, observing 200 subjects over two years. But according to one of the present study's authors, physiologist Eric Ravussin of the Pennington Biomedical Research Center in Baton Rouge, Louisiana, there are already hints that calorie restriction affects ageing processes in cells.

Participants on restricted diets lost weight exponentially, and were still losing weight after six months. They had reduced insulin resistance and reduced levels of low-density lipoprotein cholesterol, high levels of which are risk factors for type 2 diabetes and heart 
disease, respectively. The researchers also measured significantly reduced damage to volunteers' DNA. "We asked if there is a metabolic adaptation over and above what is expected due to the weight loss," says Ravussin, "and the answer is yes, there is."

$\mathrm{He}$ says the findings are consistent with the theory that calorie restriction reduces metabolic rate and lowers the production of harmful molecules called reactive oxygen species, also known as free radicals. These strip electrons from other molecules in the body; this is thought to harm cells and contribute to ageing. This metabolic change, Ravussin believes, could be what causes calorie-restricted rodents to live up to $40 \%$ longer than their unrestricted counterparts.

Clearly it will be a long wait before the CALERIE study reveals a similar effect in humans, if it ever does. Meanwhile, the research community is divided over whether calorie restriction will extend lifespan in humans.

The most recent online edition of the journal Biogerontology asked a number of experts to address this question ${ }^{3}$, in response to a new theory of longevity championed by mathematical biologist Lloyd Demetrius of Harvard University. Demetrius argues that the main factor determining lifespan is not the rate of free-radical production, but the cells' ability to resist short-term fluctuations in critical metabolites caused by environmental stresses, so protecting those cells from damage.

\section{Long haul}

Demetrius believes this is determined by a creature's evolutionary history. Rodents are opportunistic species that experience periods of plenty punctuated by periods of scarcity. When there is lots of food they reproduce like crazy; when there isn't, they sit tight. They reach sexual maturity early, have a narrow reproductive window and large litters. Humans, and larger-bodied animals in general, mature late, have fewer offspring and a wider reproductive window. They are more able to move from resource-poor regions to resource-rich ones, and are more stable in the face of environmental perturbations ${ }^{4}$.

Demetrius's theory predicts that calorie restriction would extend lifespan in opportunistic species, giving them a chance to breed once conditions improve, but barely affect lifespan in more stable species. Some evidence supports this. As Linda Partridge of University College London's Centre for Research on Ageing notes: "Extension of lifespan by dietary restriction has been seen particularly clearly in animals with high reproductive rates rodents, worms, flies." In contrast, experiments in rhesus monkeys have yet to show that calorie restriction increases lifespan ${ }^{5}$.

Believers in calorie restriction claim this is a methodological problem: monkeys live longer than rats, so the experiments take longer. Studies with rhesus monkeys begun in the United States in the late 1980s will not produce robust

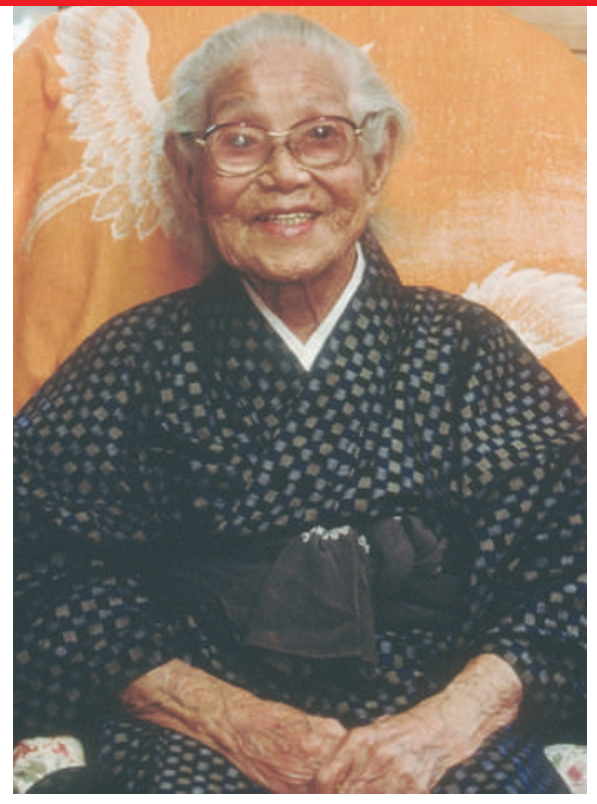

Longevity island: Oto Yara, one of Okinawa's unusually large number of centenarians.

data for another 25 years, and human studies such as CALERIE will take even longer. In the meantime, they argue, the data from studies such as CALERIE support the idea that it could indeed work in people.

Such optimism means that the development of drugs aiming to mimic calorie restriction is well advanced, with some compounds already in clinical trials ${ }^{6}$. These drugs are designed to slow ageing and possibly extend life by triggering metabolic adaptation without the need for semi-starvation. Whether or not calorie restriction increases longevity, it does seem to ward off certain age-related diseases. For example, epidemiological studies have shown that low-calorie diets are associated with lower incidences of age-related ailments such as cancer and neurodegenerative diseases ${ }^{7}$.

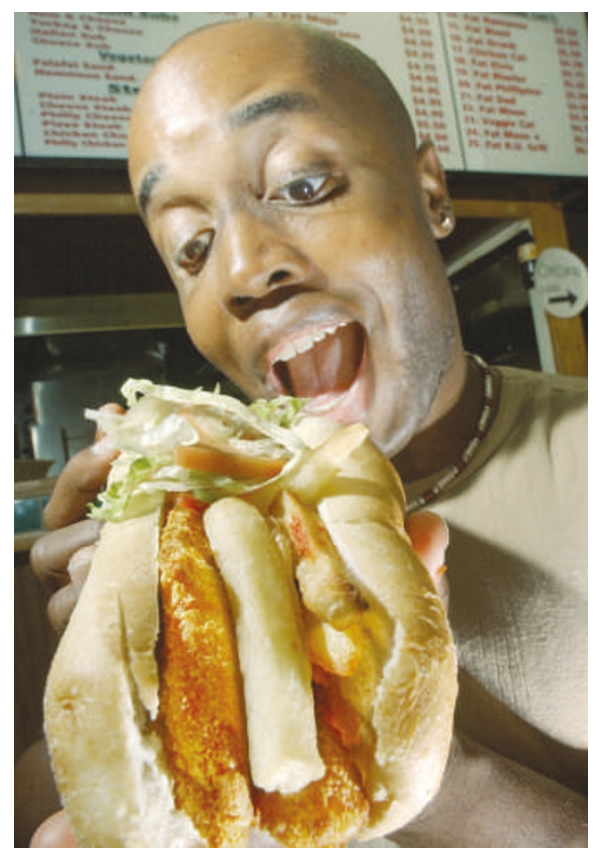

Weighty issue: separating the benefits of calorie restriction from the dangers of obesity is tricky.
The field grew out of a change in thinking about ageing. Until about 15 years ago, ageing was thought to be an unregulated process resulting from the accumulation of cellular damage. Then genetic studies, particularly in the nematode worm Caenorhabditis elegans, showed that manipulating single genes could increase or decrease lifespan dramatically ${ }^{8}$. Later, lifespan-extending mutations were found in other organisms, including the fruitfly Drosophila and rodents.

Many genes have been identified that are thought to play a part in determining lifespan, such as those involved in insulin signalling and stress resistance. One family of enzymes currently in the limelight is the sirtuins. The gene SIR2, after which the family is named, was one of the first longevity genes to be identified. Species from yeast to humans carry variants of it, and extra copies increase lifespan in yeast, worms and flies?.

\section{Sirtuin something}

Nobody knows how sirtuins might extend lifespan, but David Sinclair, who studies ageing at Harvard Medical School in Boston believes that they stabilize DNA and counteract the reduced fidelity of DNA-copying mechanisms in old cells. The Sir2 protein seems to work with a small molecule called NAD, which is involved in metabolism ${ }^{9}$, so the two together potentially explain the association between calorie restriction and ageing.

In 2003, Sinclair's team described 19 plantderived molecules that activate sirtuins in yeast ${ }^{10}$. One of these, resveratrol, found in grape skins, has been reported to have anticancer and neuroprotective effects. The chemical's presence in wine has been touted as the explanation of the 'French paradox' - the low incidence of heart disease in southern Europe, despite a fatty diet. Sinclair and others believe it may also extend life.

Working with Rafael de Cabo, Donald Ingram and others at the NIA's Laboratory of Experimental Gerontology in Baltimore, Sinclair is studying the effects of resveratrol in non-obese mice. The study's results are not yet published, but de Cabo claims they look promising. There is already published evidence that resveratrol extends life in another vertebrate. In February this year, Alessandro Cellerino and his colleagues at the Scuola Normale Superiore in Pisa, Italy, revealed that the chemical not only extends the maximum lifespan of a short-lived fish called Nothobranchius furzeri by $60 \%$, but also seems to protect the fish from neurodegeneration ${ }^{11}$.

Cynthia Kenyon, a researcher in ageing at the University of California, San Francisco, is "very impressed" with the resveratrol results, but, she points out, there is confusion over what it actually does. "In animals, at least in some cases, it looks like the effects you see are dependent on Sir2," she says. On the other hand, she says, resveratrol has so far shown no effect on Sir2 function in human cells in culture. This makes 
